\section{Caffeine improves text reading and global perception}

\author{
Sandro Franceschini ${ }^{1}$ D, Matteo Lulli ${ }^{2}$, Sara Bertoni ${ }^{1}$, \\ Simone Gori ${ }^{3}$, Alessandro Angrilli ${ }^{1}$, Martina Mancarella ${ }^{1}$, \\ Giovanna Puccio ${ }^{1}$ and Andrea Facoetti ${ }^{1}$
}

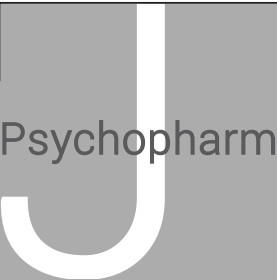

Journal of Psychopharmacology 1-11

(c) The Author(s) 2019 Article reuse guidelines: sagepub.com/journals-permissions DOI: $10.1177 / 0269881119878178$ journals.sagepub.com/home/jop

(\$)SAGE

\begin{abstract}
Background: Reading is a unique human skill. Several brain networks involved in this complex skill mainly involve the left hemisphere language areas. Nevertheless, nonlinguistic networks found in the right hemisphere also seem to be involved in sentence and text reading. These areas do not deal with phonological information, but are involved in verbal and nonverbal pattern information processing. The right hemisphere is responsible for global processing of a scene, which is needed for developing reading skills.

Aims: Caffeine seems to affect global pattern processing specifically. Consequently, our aim was to discover if it could enhance text reading skill. Methods: In two mechanistic studies ( $n=24$ and $n=53$ ), we tested several reading skills, global and local perception, alerting, spatial attention and executive functions, as well as rapid automatised naming and phonological memory, using a double-blind, within-subjects, repeated-measures design in typical young adult readers.

Results: A single dose of 200 mg caffeine improved global processing, without any effect on local information processing, alerting, spatial attention and executive or phonological functions. This improvement in global processing was accompanied by faster text reading speed of meaningful sentences, whereas single word/pseudoword or pseudoword text reading abilities were not affected. These effects of caffeine on reading ability were enhanced by mild sleep deprivation.
\end{abstract}

Conclusions: These findings show that a small quantity of caffeine could improve global processing and text reading skills in adults.

\title{
Keywords
}

Visual perception, reading enhancement, parallel processing, psychostimulant, context processing

\section{Introduction}

Reading is a unique human skill practiced by millions of people daily. Reading achievement is dependent on several cognitive skills. In particular, visual-orthographic and auditory-phonological left brain networks and their connections (see Dehaene et al., 2015 for a review), which are also employed for other daily activities, contribute to the acquisition of reading skills (e.g. Carroll et al., 2016; Franceschini et al., 2017; see Hancock et al., 2017 for a recent review). The first analysis of a written word or a sentence is visual. Visual stimuli are processed hierarchically: the right hemisphere is responsible for the first global perception of the visual scene, whereas the left hemisphere underlies the later local perception of detail in the stimuli (e.g. Delis et al., 1986; Martinez et al., 1997; Sergent, 1982; Wiesmman and Banich, 1999; Yamaguchi et al., 2000; see for a review Kauffmann et al., 2014). This hierarchical analysis is driven by the fronto-parietal attentional network (Wiesmman et al., 2006; Wiesmman and Woldoroff, 2004), and linked to occipital visual pathway functioning (Proverbio et al., 1998), and it is an essential component of our visual system (Navon, 1977). Consequently, a so-called 'Navon figure', a large circle composed of small squares, will first be perceived as a circle and not be seen as multiple small squares (e.g. see Figure 1).

During reading acquisition, this right-to-left hemisphere perceptual sequence probably plays a pivotal role. Analysis of the global pattern, before local analysis of the single letters inside a word, as well as of sentence shape before the local analysis of a single word in the text, may rapidly narrow down the range of candidates in a local region and enable assignment of their correct location (Hochstein and Ahissar, 2002).

The right hemisphere does not host phonological information (Vigneau et al., 2011). However, only during sentence/text processing, related to context processing, has specific right hemisphere involvement been described, but not during lexical or sublexical processing (Vigneau et al., 2011). Horowitz-Kraus et al. (2014) showed that in young adults, activation of the right inferior frontal gyrus and the structural connectivity of the right fasciculus arcuatus are specifically connected to reading comprehension of sentences, whereas the same structures were not involved in a single word reading task. Thus, they demonstrated the existence of a dissociation between the word and narrative levels of reading performance in typical readers. The structural

\footnotetext{
${ }^{1}$ Developmental and Cognitive Neuroscience Lab, Department of General Psychology, University of Padua, Padua, Italy

${ }^{2}$ Department of Experimental and Clinical Biomedical Sciences 'Mario

Serio', University of Florence, Florence, Italy

${ }^{3}$ Department of Human and Social Sciences, University of Bergamo, Bergamo, Italy
}

\section{Corresponding author:}

Sandro Franceschini, Developmental and Cognitive Neuroscience Lab, Department of General Psychology, University of Padua, Via Venezia 8, Padua, 35131, Italy.

Email: sandro.franceschini@unipd.it 


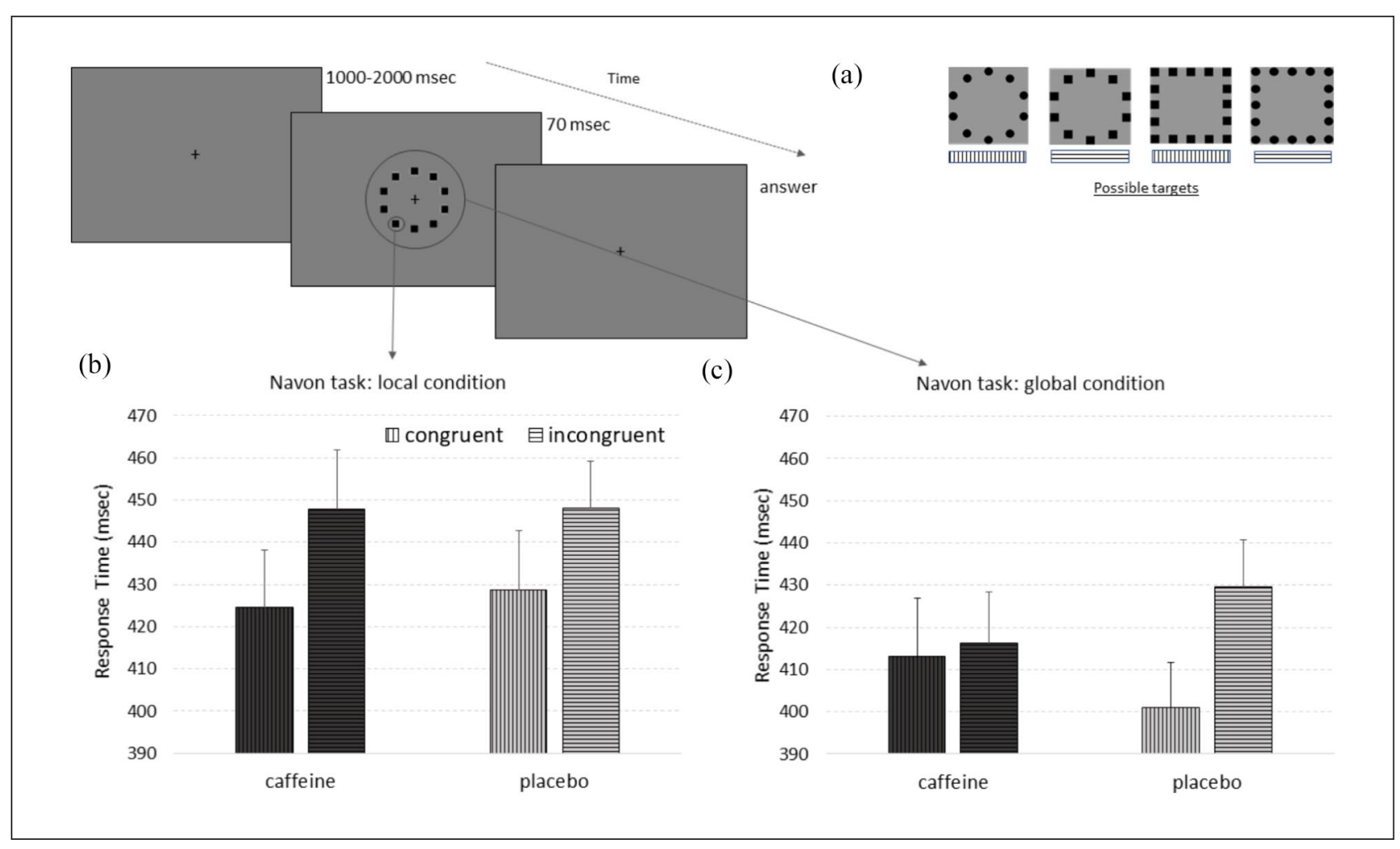

Figure 1. Computerised Navon task. (a) Description of the Navon tasks (global and local). Participants - using two buttons on computer keyboard - had to indicate whether the global (or local) figure was a square or a circle, independently from local (or global) figures. (b) In the local task, incongruent targets need more time to be discriminated independently from caffeine or placebo intake. (c) In the global task incongruent targets need more time to be discriminated only after placebo intake, whereas after caffeine intake the difference between congruent and incongruent condition disappear. Error bars represent mean standard errors.

connectivity of the same areas of the right hemisphere strongly predicted narrative comprehension, evaluated using a sentencepicture matching task (Horowitz-Kraus et al., 2015). This right fronto-parietal network involvement in lexico-semantic tasks probably enables the deployment of additional attentional resources to manipulate information retrieved from working memory (Vigneau et al., 2011; Zago et al., 2008). Single-pulse transcranial magnetic stimulation to the right, but not the left, frontal eye field, which is strongly linked to the posterior parietal cortex, is indeed able to interfere with the deployment of attentional resources (Ronconi et al., 2012).

Consistent with a possible involvement of global processing, controlled by the right hemisphere, in reading efficiency, Franceschini et al. (2017) showed a specific deficit in global perception in children with developmental dyslexia. In particular, two different remediation programmes - one based on time-constrained sentence reading (Breznitz et al., 2013) and one based on action video games (see Peters et al., 2019 for a recent review) were able to improve both global perception in a Navon task and reading efficiency (Franceschini et al., 2017).

Hoeft et al. (2011), studying the neural systems involved in remediating reading difficulties, showed that the right inferior frontal gyrus and the right fasciculus arcuatus are critical areas for reading improvements in children with developmental dyslexia. Moreover, in a prospective longitudinal study evaluating a large group of pre-reading children in the last year of kindergarten, the development of hierarchical global-to-local processing in a Navon task specifically predicted their future text reading abilities in the first grade of primary school (Franceschini et al., 2017). Together, these studies show that the right frontoparietal areas are involved in the information processing of complex patterns such as text.

Caffeine (1,3,7-trimethylxanthine) is one of the most widely consumed psychoactive substances (Einöther and Giesbrecht, 2013; Ferré, 2008; Heckman et al., 2010; Smith, 2002). It seems to affect the nervous system by inhibiting the binding of adenosine and benzodiazepine to receptors on nerve membranes, thus reducing the normal inhibitory effects of these ligands. However, caffeine also affects many other neurotransmitter systems, including noradrenaline, dopamine, serotonin, acetylcholine, glutamate and gamma-aminobutyric acid (Fredholm et al., 1999).

Meta-analyses of several double-blind studies has confirmed that caffeine improves physical exercise performance, probably by reducing feelings of exhaustion (Doherty and Smith, 2005; Ruxton, 2008). It also appears to counter both the physical and cognitive effects of sleep loss (McLellan et al., 2016), and these effects are larger the less the sleep debt (Linde, 1994). It does more than merely restore performance degraded by sleep deprivation, muscular fatigue, or boredom (Weiss and Laties, 1962); beneficial effects of caffeine have been observed not only in simple reaction times for stimulus detection but also in more complex cognitive tasks, in which active monitoring and coordination of 
Table 1. Descriptive statistics: age, mean daily caffeine intake, performance in standardised reading tasks mean (and standard deviation) of the two groups of participants in the first and second study.

\begin{tabular}{lcc}
\hline & First study $n=24$ & $\begin{array}{c}\text { Second study } n=53 \\
\text { Mean (SD) }\end{array}$ \\
\hline Age mean (years) & $25(4.7)$ & $21(1.66)$ \\
Caffeine daily intakes average $(\mathrm{mg})$ & $103(87)$ & $111(56)$ \\
Word list reading speed $(Z$ score) & $-0.44(0.92)$ & $-0.62(0.93)$ \\
Word list reading accuracy (Z score) & $-0.42(1.32)$ & $0.06(1.03)$ \\
Pseudo-word list reading speed (Z score) & $0.08(1.13)$ & $0.04(0.97)$ \\
Pseudo-word list reading accuracy (Z score) & $-0.20(1.05)$ & $-0.42(1.29)$ \\
\hline
\end{tabular}

SD: standard deviation.

behaviour are necessary (e.g. in choice reaction and rapid visual information processing tasks; see Einöther and Giesbrecht, 2013; Nehlig, 2004). Mixed results have been observed for the effects of caffeine on short-term memory, in particular for verbal and written materials (McLellan et al., 2016). Cognitive tasks that involve abilities like arithmetic, writing speed and memorization of written materials were not significantly affected by caffeine (Nehlig, 2004; Weiss and Laties, 1962). Interestingly, in narrative comprehension tasks where the participant has to read a sentence and to evaluate whether the represented picture is correct, caffeine was found to improve speed and accuracy (Nehlig, 2004).

Caffeine seems to facilitate the identification of the global pattern in a Navon figure without changing identification of the local components (Mahoney et al., 2011). Moreover, in line with research on logical reasoning (Nehlig, 2004), caffeine seems to enhance the global pattern analysis of sentences, improving the detection of morphosyntactic violations during a proofreading task, but leaving unaffected the ability to detect misspelled words (Brunyé et al., 2012).

Based on these studies, we postulated that caffeine, through its effects on global perception, logical reasoning and lexicosemantic tasks, might also have beneficial effects on reading tasks. Testing typical young adult readers, we expected that caffeine would affect text reading with no effects on other reading skills or cognitive functions related to reading, such as working memory or phonological skills (Brunyé et al., 2012; Franceschini et al., 2017; Horowitz-Kraus et al., 2014; Vigneau et al., 2011).

A first study was conducted to test for beneficial effects of caffeine on reading and to estimate their effect size. To elucidate the specific connections between different attentional-perceptual skills and reading enhancement, we conducted a second study.

\section{Materials and methods}

A double-blind, repeated-measures design was used in two mechanistic studies. The goal of a mechanistic study is to identify the mechanism(s) of action of an intervention on cognitive enhancement. In other words, the question is not whether, but how. Mechanistic studies test an explicit hypothesis, generated by a clear theoretical framework, about the mechanism of action of a particular cognitive enhancement (Green et al., 2019). The estimation of the effect size of caffeine on reading skills measured in the first study was used to define the sample size for the second replication study. Caffeine's bitter taste was masked by using a bitter drink, thus allowing a real double-blind design (see
Supplementary Material). The order of administration of caffeine or placebo solutions was counterbalanced $(200 \mathrm{mg}$ caffeine or placebo; placebo or $200 \mathrm{mg}$ caffeine; about two espressos) across participants. The entire investigation process was conducted according to the principles expressed in the Declaration of Helsinki. Written informed consent was obtained from all subjects, and all procedures were jointly approved by the HIT Ethics Committee of the University of Padua.

\section{Participants}

The first study involved 24 volunteers (18 female) who were low and normal habitual caffeine consumers (Barone and Roberts, 1996; see Table 1). Participants were tested twice at 1-7 days apart. Evaluations were scheduled in the morning, at the same hours (also see Supplementary Material).

Fifty-three low-to-normal habitual caffeine consumers (41 female; see Table 1) participated in the second replication study. Participants were tested twice at seven days apart, using the same procedural design of the first study. For each participant, a saliva sample was collected $30 \mathrm{~min}$ after the beverage intake (at the beginning of the administration of tasks; see Supplementary Material).

All the participants were invited to attend two meeting sessions. They were asked, before each session, to avoid consuming foods containing caffeine for $12 \mathrm{~h}$ and to sleep a number of hours adapted to their habits. At the beginning of the experimental session, each participant was asked how many hours she/he had slept. This information was used to calculate the slept debt (placebo minus caffeine session). The participants were aware that in one of the two sessions they would have to drink a beverage containing caffeine, and in the other they would drink the same beverage without caffeine.

\section{Procedures and methods}

\section{Reading and reading-related abilities evaluations}

Standardised reading test. We measured the reading levels of the participants using standardised word and pseudoword lists reading test (Stella and Tintoni, 2007) at the first experimental session before the administration of caffeine or placebo. See Table 1 for descriptive statistics, as well as the Supplementary Material.

All the tasks described below were administered in a randomised order. 
Word text reading. Text reading abilities were evaluated using two texts of similar length and reading difficulty (Judica and De Luca, 1993). Reading speed (syllables per second) and number of errors were measured.

The two texts were administered in counterbalanced order in the two sessions between the different participants. Participants were invited to read each text aloud as quickly and as accurately as possible. A word that was wrongly read was counted as one error independently from the quantity of wrong letters or syllables pronounced. Self-correction was counted as a 0.5 error.

Pseudoword text reading. Phonological decoding ability was measured using two texts, each of 46 pseudowords composed of 1-3 syllables (same syllables in different order for both texts) for a total of 100 syllables for each text (Franceschini et al., 2016). The two texts were administered in counterbalanced order in the two sessions between the different participants. Reading speed (syllables per second) and the number of errors were measured. All participants were invited to read each text aloud as quickly and as accurately as possible. A pseudoword that was wrongly read was counted as one error independently from the quantity of wrong letters or syllables pronounced. Self-correction was not classified as error.

Lexical long-term memory. Two texts of similar length and complexity (Costanzo et al., 2012) were administered in randomised and counterbalanced order in the two sessions. After (silently) reading the text without time limits, the participants were invited to answer ten questions concerning particular contents in the text just read.

Two examples of questions:

1. How are the leaves? A. Wide and shiny; B. Thin and shiny; C. Long and shiny; D. Wide and bright;

2. What's near the plant? A. Curtain and umbrella stand; B. Tent and watering can; C. Watering can and umbrella stand; D. Curtain and another vase.

Rapid automatised naming. Two lists of 30 colour-filled squares (red, green, yellow, and blue; six rows of five squares each) were presented to participants in counterbalanced and randomised order on an A4 sheet of paper. Participants were invited to name aloud each colour as quickly and accurately as possible. Response time was measured in seconds.

Anxiety evaluation. We used a 20-item questionnaire adapted from the State-Trait Anxiety Inventory (Lazzari and Panchieri, 1980) to evaluate the state of anxiety. The total score was measured and analysed.

\section{Second study: Additional procedures and methods}

Computerised reading tasks. The experimental procedure and data acquisition for the computerised tasks were controlled using E-prime 2.0 (Psychology Software, Inc.) running on an 18-inch screen. The viewing distance was set to $50 \mathrm{~cm}$.
Single word reading task. Two lists of 72 words, paired for number of letters (6-9) and use frequency (List 1 mean=123, standard deviation $(\mathrm{SD})=183$; List 2 mean=123.5, $\mathrm{SD}=178$ ), were used. The two lists were administered in counterbalanced order in the two sessions between the different subjects. Participants were instructed to keep their eyes on the fixation point $\left(0.1^{\circ}\right.$ and $0.6 \mathrm{~cd} /$ $\mathrm{m}^{2}$ ) for the duration of the trial. A black cross fixation appeared on a grey computer screen $\left(40 \mathrm{~cd} / \mathrm{m}^{2}\right)$. After a randomised quantity of time (1500-2000 ms), one word (colour=black, font=Courier New, size $=18,0.4^{\circ}$ for each letter) appeared at the centre of the screen. The target word remained on the screen until the participant answered. When the microphone was activated, the word was replaced by a mask. Vocal response time (in ms) and accuracy of each single response were recorded. Words that were wrongly read were counted as one error independently from the quantity of wrong letters or syllables pronounced. Five training trials were proposed before the experimental session.

Single pseudoword reading task. Two lists of 36 pseudowords, paired for number of letters (6-9), were used. Pseudowords were made up starting from the word reading task lists, inverting syllables and in some cases changing syllables between the two lists. The administration procedure was the same as the single word reading task. Vocal response time (in ms) and accuracy of single responses were recorded. Five training trials were proposed before the experimental session.

Perceptual Navon task. Global and local Navon tasks were administered in counterbalanced order within and between participants (see Franceschini et al., 2017 for details). Geometric figures were shown on a computer screen: a square or a circle $\left(9.5^{\circ} \times 9.5^{\circ}\right)$ at a global level, which could be formed by small squares or circles $\left(1.3^{\circ} \times 1.3^{\circ}\right)$ at the local level (see Figure 1(a)).

Global task: the participant had to indicate the global figure pressing one of two keyboard buttons. Stimuli features were either congruent or incongruent: in the congruent condition the global figure had the same shape as local figures (a big square composed by little blue squares, $8 \mathrm{~cd} / \mathrm{m}^{2}$ ), whereas in the incongruent condition the global figure had a different shape from local figures (a big circle composed by little blue squares). Response time (in ms) and accuracy of single responses were recorded. The accuracy rate was at ceiling (greater than 0.95 in all the conditions), and consequently was not analysed.

Local task: the local Navon task had exactly the same structure as the global Navon task. In this case, a participant had to indicate the local figures. Response time (in ms) and accuracy of single responses were recorded. The accuracy rate was at ceiling (greater than 0.95 in all the conditions), and consequently was not analysed.

Attentional Network Task. Participants were instructed to keep their eyes on the fixation point for the duration of the trial (see Figure 2(a)). Each trial started with a grey display $\left(40 \mathrm{~cd} / \mathrm{m}^{2}\right)$ and the black fixation point $\left(2000 \mathrm{~ms}\right.$ and $\left.0.6 \mathrm{~cd} / \mathrm{m}^{2}\right)$. One of four possible cue conditions (no cue, double cues, valid or invalid cue with $50 \%$ of predictivity) appeared for $50 \mathrm{~ms}$. In the no cue condition, only the 


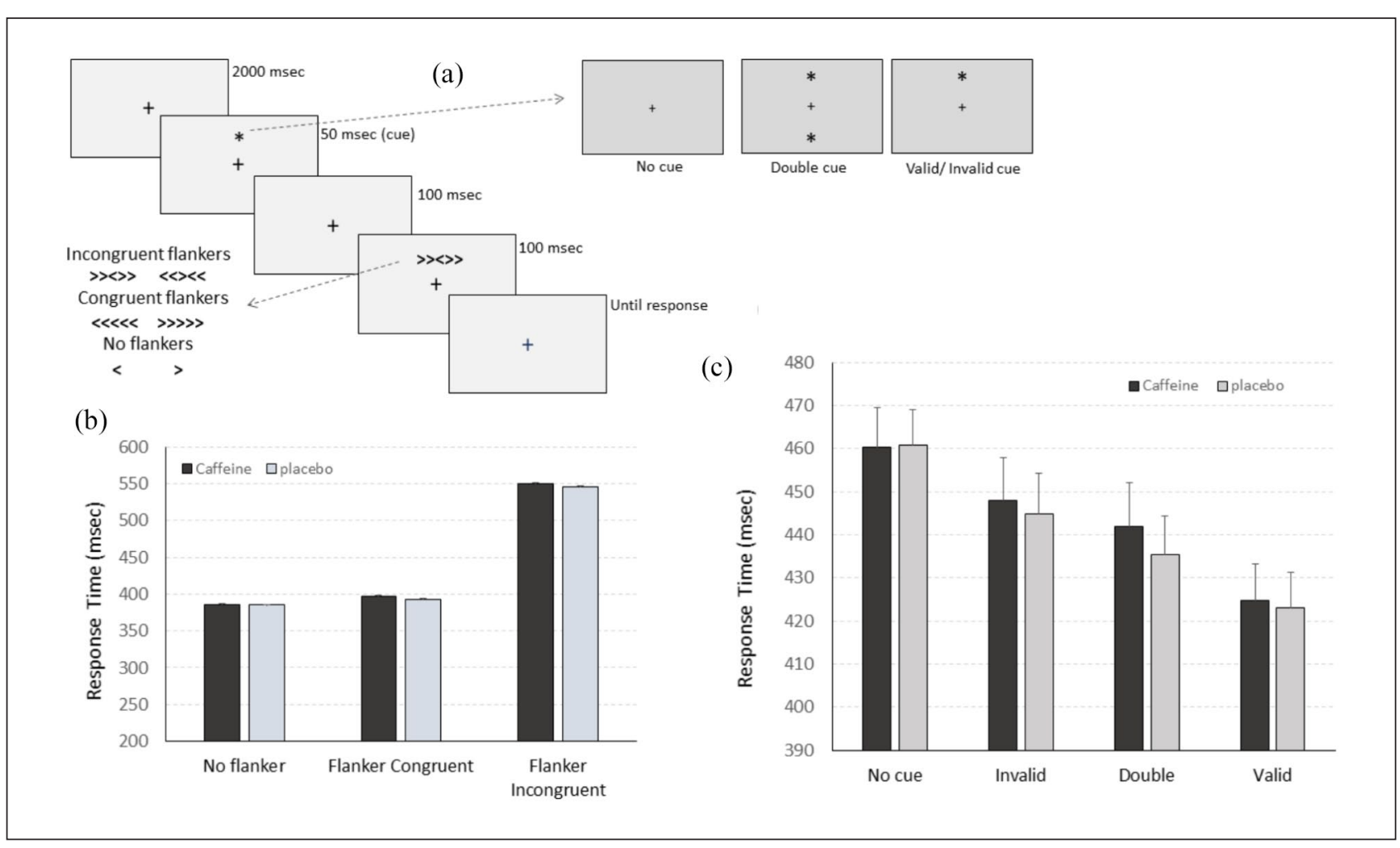

Figure 2. Attentional Network Task. (a) Description of the Attentional Network Task. Participants, using two buttons on computer keyboard, had to indicate whether the central arrowhead (that could appear isolated or flanked by congruent or incongruent arrowheads) was oriented towards left or right. The target could be preceded by valid (an asterisk in the same position of the target), invalid (opposite position), double cue (both positions) or by fixation point only (no cue condition). (b) Response time (in ms) after caffeine and placebo intake in presence of no flankers, congruent and incongruent flankers. Comparing caffeine and placebo response time, all $p s$ were greater than 0.56 ; Cohen's $d=0.08,95 \%$ confidence interval $(C I)=-0.19-35$; Bayesian factor $(B F)_{01}=5.677$. (c) Response time (in ms) after the four different cue conditions. Comparing caffeine and placebo response time, all $p$ s were greater than 0.458 , Cohen's $d=0.03295 \% \mathrm{CI}=-0.237-301 ; \mathrm{BF}_{01}=5.117$. Error bars represent mean standard error.

fixation point was presented. In the valid cue condition, an asterisk $\left(1^{\circ}\right.$ and $\left.0.6 \mathrm{~cd} / \mathrm{m}^{2}\right)$ appeared above or below $\left(3.4^{\circ}\right)$ the fixation point $\left(0.5^{\circ}\right.$ and $\left.0.6 \mathrm{~cd} / \mathrm{m}^{2}\right)$, in the same position where the target appeared, whereas in the invalid cue condition, the asterisk appeared in the opposite location of the target. In the case of double cues, two asterisks appeared together in both possible locations of the target. After $100 \mathrm{~ms}$ with only the fixation point presented on the screen, the target appeared for $100 \mathrm{~ms}$. The target was an arrowhead oriented right or left $\left(1^{\circ}\right.$ and $\left.0.6 \mathrm{~cd} / \mathrm{m}^{2}\right)$ that could appear alone or flanked by four arrowheads $\left(5.7^{\circ}\right.$ the four distractors and the central target) oriented in congruent or incongruent direction with the target. As soon as the target disappeared, the fixation point became blue $\left(1 \mathrm{~cd} / \mathrm{m}^{2}\right)$. The participant could indicate as quickly as possible the direction of the target, pressing one of two keys ( $\mathrm{Z}$ or $\mathrm{M})$ on the computer keyboard. The experimental session was composed of 480 trials, plus eight trials used in the training session. Response time (in ms) and accuracy of single responses were recorded. Accuracy was at ceiling in both sessions (caffeine mean rate $=0.94, \mathrm{SD}=0.07$; placebo $=0.93$, $\mathrm{SD}=0.09$ ), and consequently not analysed.

\section{Phonological short-term memory}

Participants listened to a series of pseudoword trigrams. Participants had to repeat each trigram in the correct sequence. A couple of trigrams were presented. If a participant repeated at least one of them correctly, then a new couple with an additional trigram was proposed. If both lists were wrongly reported, then the task was interrupted. One point for each correctly repeated phoneme was assigned. The series started with two trigrams in each couple and continued up to a maximum of eight trigrams. The total number of correct phonemes was measured. Trigrams were different (counterbalanced) in the two evaluations.

\section{Results}

\section{Reading skills}

In the first study, a comparison of reading speed measured in syllables per second (syll/s) on two reading word texts showed that consumption of a single dose of $200 \mathrm{mg}$ of caffeine significantly improved reading speed (mean $=5.8, \mathrm{SD}=0.84$ ) compared to placebo consumption condition (mean $=5.69, \mathrm{SD}=0.75 ; t=1.911$, $p=0.035$, one-tailed), without significantly affecting the number of errors (see Table 2).

The presence of a beneficial effect of caffeine on word text reading speed was confirmed in the second replication study. Analysis of text reading performance established that after caffeine intake, participants significantly improved their reading 
Table 2. Results summary table. Performance in reading, verbal memory tasks, the state anxiety questionnaire and hours of sleep before experimental session of participants in the first and second study.

\begin{tabular}{|c|c|c|c|c|c|}
\hline First study $(n=24)$ & Caffeine mean (SD) & Placebo mean (SD) & $t$ Test and $p$ value & Cohen's $d$ and 95\% CI & $\mathrm{BF}$ \\
\hline Word text (syll/s) & $5.80(0.84)$ & $5.69(0.75)$ & $\begin{array}{l}t_{(23)}=1.911, p=0.035 \\
\text { one tailed }\end{array}$ & $\begin{array}{l}0.39 \\
-0.029-0.802\end{array}$ & $\mathrm{BF}_{10}=1.017$ \\
\hline Word text errors (number) & $8.21(6.08)$ & $9.15(4.52)$ & $t_{(23)}=-0.839, p=0.41$ & $\begin{array}{l}-0.171 \\
-0.572-0.234\end{array}$ & $\mathrm{BF}_{01}=3.391$ \\
\hline Pseudoword text (syll/s) & $3.42(0.64)$ & $3.41(0.65)$ & $t_{(23)}=0.107, p=0.916$ & $\begin{array}{l}0.022 \\
-0.379-0.422\end{array}$ & $\mathrm{BF}_{01}=4.634$ \\
\hline Pseudoword text errors (number) & $2.54(2.38)$ & $2.71(1.81)$ & $t_{(23)}=-0.368, p=0.716$ & $\begin{array}{l}-0.075 \\
-0.475-0.326\end{array}$ & $\mathrm{BF}_{01}=4.379$ \\
\hline Lexical long term memory (correct) & $5.42(1.44)$ & $5.42(1.59)$ & $t_{(23)}<0.0001, p=0.999$ & $\begin{array}{l}0 \\
-0.566-0.566\end{array}$ & $\mathrm{BF}_{01}>30$ \\
\hline Rapid Automatised Naming (s) & $15.98(2.76)$ & $16.51(2.58)$ & $t_{(23)=1.313, p=0.202}$ & $\begin{array}{l}-0.268 \\
-0.673-0.142\end{array}$ & $\mathrm{BF}_{01}=2.175$ \\
\hline Anxiety (STAI raw score) & $36.04(8.09)$ & $34.96(7.92)$ & $t_{(23)}<0.865, p=0.396$ & $\begin{array}{l}0.177 \\
-0.229-0.578\end{array}$ & $\mathrm{BF}_{01}=3.324$ \\
\hline Hours of sleep before experimental sessions & $7.41(0.61)$ & $7.18(1.14)$ & $t_{(23)}=1.038, p=0.31$ & $\begin{array}{l}0.212 \\
-0.195-0.614\end{array}$ & $\mathrm{BF}_{01}=2.874$ \\
\hline \multicolumn{6}{|l|}{ Second study $(n=53)$} \\
\hline Word text (syll/s) & $5.90(0.92)$ & $5.81(0.88)$ & $t_{(52)}=2.139, p=0.037$ & $\begin{array}{l}0.294 \\
0.017-0.568\end{array}$ & $\mathrm{BF}_{10}=1.216$ \\
\hline Word text errors (number) & $8.25(4.27)$ & $8.27(4.79)$ & $t_{(52)}=-0.041, p=0.968$ & $\begin{array}{l}-0.006 \\
-0.275-0.265\end{array}$ & $\mathrm{BF}_{01}=6.673$ \\
\hline Pseudoword text (syll/s) & $3.61(0.72)$ & $3.68(0.71)$ & $t_{(52)}=-0.808, p=0.423$ & $\begin{array}{l}-0.111 \\
-0.38-0.16\end{array}$ & $\mathrm{BF}_{01}=4.903$ \\
\hline Pseudoword text errors (number) & $2.53(1.85)$ & $2.98(2.38)$ & $t_{(52)}=-1.25, p=0.217$ & $\begin{array}{l}-0.172 \\
-0.442-0.1\end{array}$ & $\mathrm{BF}_{01}=3.2$ \\
\hline Single word (ms) & $515(84)$ & $521(76)$ & $t_{(52)}=0.908, p=0.368$ & $\begin{array}{l}-0.125 \\
-0.394-0.146\end{array}$ & $\mathrm{BF}_{01}=4.521$ \\
\hline Single word accuracy (rate) & $0.99(0.02)$ & $98(0.02)$ & $t_{(52)}=1.061, p=0.293$ & $\begin{array}{l}0.146 \\
-0.126-0.416\end{array}$ & $\mathrm{BF}_{01}=3.925$ \\
\hline Single pseudoword (ms) & $774(165)$ & $774(160)$ & $t_{(52)}=-0.027 p=0.978$ & $\begin{array}{l}-0.004 \\
-0.273-0.266\end{array}$ & $\mathrm{BF}_{01}=6.676$ \\
\hline Single pseudoword accuracy (rate) & $0.85(0.10)$ & $0.86(0.09)$ & $t_{(52)}=1.193, p=0.238$ & $\begin{array}{l}-0.164 \\
-0.434-0.108\end{array}$ & $\mathrm{BF}_{01}=3.418$ \\
\hline Rapid Automatised Naming (s) & $15(2.81)$ & $15(2.97)$ & $t_{(52)}=-1.156, p=0.253$ & $\begin{array}{l}-0.159 \\
-0.429-0.113\end{array}$ & $\mathrm{BF}_{01}=3.561$ \\
\hline Phono short-term memory ( $n$ phonemes) & $50(17)$ & $51(15)$ & $t_{(52)}=-0.389, p=0.699$ & $\begin{array}{l}-0.053 \\
-0.323-0.216\end{array}$ & $\mathrm{BF}_{01}=6.214$ \\
\hline Lexical long-term memory (correct answers) & $5.75(1.62)$ & $5.57(1.58)$ & $t_{(52)}=0.629, p=0.532$ & $\begin{array}{l}0.086 \\
-0.184-0.356\end{array}$ & $\mathrm{BF}_{01}=5.535$ \\
\hline Anxiety (STAI raw score) & $34.51(6.93)$ & $35.85(6.02)$ & $t_{(52)=}-1.51, p=0.136$ & $\begin{array}{l}-0.208 \\
-0.479-0.065\end{array}$ & $\mathrm{BF}_{01}=2.29$ \\
\hline Hours of sleep before experimental sessions & $7.16(1.29)$ & $7.05(1.36)$ & $t_{(52)}=0.615, p=0.541$ & $\begin{array}{l}0.084 \\
-0.186-0.354\end{array}$ & $\mathrm{BF}_{01}=5.58$ \\
\hline
\end{tabular}

CI: confidence interval; SD: standard deviation.

speed $(\mathrm{syll} / \mathrm{s}=5.90 \mathrm{SD}=0.92)$, in comparison to the placebo condition $\left(\mathrm{syll} / \mathrm{s}=5.81, \mathrm{SD}=0.88, t_{(52)}=2.139, p=0.037\right)$, without significantly affecting the number of errors (see Table 2$)$.

A $t$-test on the entire sample of participants $(24+53=77$ participants) showed a significant difference in text reading speed (caffeine mean syll $/ \mathrm{s}=5.87, \mathrm{SD}=0.89$; placebo mean=5.77, $\mathrm{SD}=0.84 ; t_{(76)}=2.843, p=0.006$, Cohen's $d=0.329,95 \%$ confidence interval $(\mathrm{CI})=0.094-0.552$; Bayesian factor $(\mathrm{BF})_{10}=5.185$; see Figure 3 and Supplementary Material). To estimate the statistical power of our data, we carried out a post-hoc power analysis ( $\alpha$ err prob: 0.05 ): power (1- $\beta$ error prob) was 0.81 (G.power; http://www.gpower.hhu.de/).

To test for possible gender difference effects of caffeine (Adan et al., 2008), we conducted an additional exploratory nonparametric analysis (Wilcoxon) on text reading speed both in female and male subsamples. In the female subsample, the difference was not significant $(Z=-1.691, p=0.091$ Cohen's $d=0.229$, $\left.95 \% \mathrm{CI}=-0.031-0.487 ; \mathrm{BF}_{10}=0.603\right)$, whereas in the male 


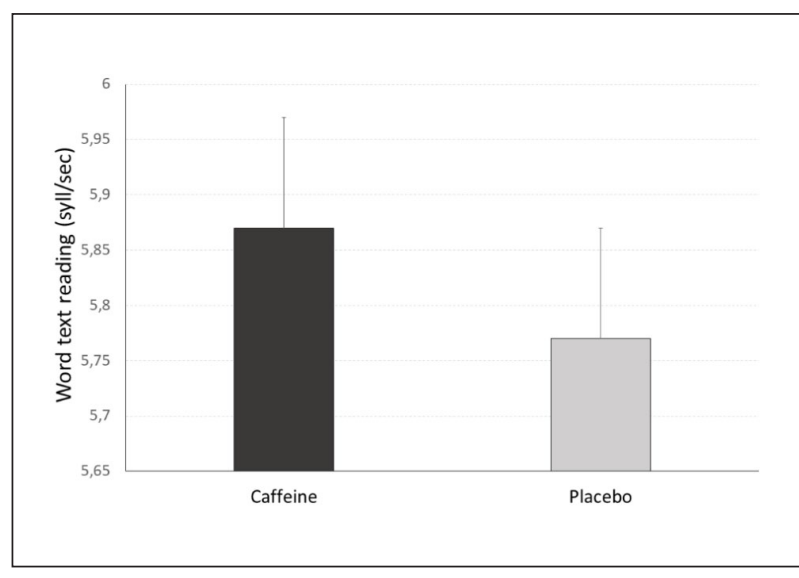

Figure 3. Text reading speed. Mean reading speed in word text reading (syllables per second) of the 77 participants of our study. The same participants read two passages of similar difficulties after caffeine or placebo intake. Caffeine significantly improved reading speed. Error bars represent mean standard error.

subsample the difference was significant $(Z=-2.069, p=0.039$ Cohen's $\left.d=0.619,95 \% \mathrm{CI}=-0.105-1.117 ; \mathrm{BF}_{10}=3.302\right)$.

The improvements consistently observed in both studies in word text reading speed were not generalised to other reading tasks. Phonological decoding speed and errors, measured with texts composed by pseudowords, as well as the access to the phonological lexicon, measured with a rapid automatised naming of colours, were not influenced by caffeine intake (see Table 2). Improvement in text reading speed did not appear to be due to a faster decoding of the single elements that compose the string of letters or a faster access to phonological lexicon.

In the second study, we also added to these tasks a more sensitive computerised evaluation of reading skills. In this task, single words or pseudowords were presented and accuracy and vocal response times were registered: no significant effect due to caffeine intake was found (all $p \mathrm{~s}>0.23$; see Table 2). Reading speed improved only when words were organised in sentences.

\section{Memory tasks}

In both studies, we tested the influence of caffeine intake on lexical long-term memory abilities. A multiple-choice questionnaire about information contained in a brief text just read (different from the one used to evaluate reading speed and accuracy) was administered. Performance was not modified by caffeine intake (see Table 2). In the second study, we also tested the phonological short-term memory (memory of trigrams): again, caffeine did not influence the number of correctly repeated phonemes. Reading improvement was not accompanied by improvement in longterm or short-term phonological memory skills.

\section{Perceptual Navon task}

We observed that caffeine intake led to better extraction of global information without the influence of local details. In the Navon global task, after placebo intake, the small (local) incongruent figures negatively influenced the choice response time of the participants $\left(t_{(52)}=-3.553, p=0.001\right.$ Cohen's $d=-0.488,95 \%$ $\left.\mathrm{CI}=-0.771--0.201 ; \mathrm{BF}_{01}=0.03\right)$. In contrast, after caffeine intake, local congruent and incongruent figures produced similar interference $\left(t_{(52)}=0.331, p=0.742\right.$; Cohen's $d=-0.046,95 \%$ $\mathrm{CI}=-0.315-0.224 ; \mathrm{BF}_{01}=0.03$; see Figure 2(c) and Supplementary Material). The incongruence effect (incongruent minus congruent response time) results were significantly different between placebo (mean=29 $\mathrm{ms} \mathrm{SD}=59)$ and caffeine (mean=3 $\mathrm{ms}, \mathrm{SD}=65$, $t_{(52)}=2.406, p=0.02$, Cohen's $d=-0.33,95 \% \mathrm{CI}=-0.605--0.052$; $\mathrm{BF}_{10}=2.058$ ).

We tested the possible difference in genders (Adan et al., 2008) in the performance in the global Navon task. Exploratory nonparametric analysis showed that in both male and female samples, in placebo conditions, the small incongruent figures negatively influenced the choice response time of the participants (female $Z=-2.754, p=0.006$, Cohen's $d=0.494,95 \% \mathrm{CI}=0.712$ $0.187 ; \mathrm{BF}_{10}=4.378$; male $Z=-2.197, p=0.028$, Cohen's $d=0.718$, $95 \% \mathrm{CI}=0.913-0.257 ; \mathrm{BF}_{10}=3.183$ ); after caffeine intake, both subsamples showed a similar interference effect from congruent and incongruent local figures (female $Z=-0.901, p=0.368$, Cohen's $d=0.161,95 \% \quad \mathrm{CI}=-0.473-0.186 \quad \mathrm{BF}_{01}=5.919$; male $Z=-0.706, p=0.48$, Cohen's $d=-0.23195 \% \mathrm{CI}=-0.704-0.385$; $\mathrm{BF}_{01}=2.196$ ).

However, the size of the incongruence effect in individuals did not predict their improvements in reading speed $(r=-0.01$, $p=0.95$ ). Consequently, to evaluate the possible nonlinear connection between global perception and reading skills, we computed the odds ratio between two categorical variables: the reduction in the incongruence effect (incongruence effect in placebo minus incongruence effect in caffeine condition, greater than zero) and an improvement in text reading speed (syllable per second in caffeine minus placebo, greater than zero). The odds ratio was 2.88 (95\% CI=0.9-9.17), indicating a moderate relation between the two variables.

It could be supposed that caffeine simply boosted the executive functions facilitating the inhibition in the incongruent condition. This hypothesis was not confirmed by the analysis in the Navon local task. In this case, executive functions played a determinant role: the global information came first and needed to be ignored to allow a rapid identification of the local information (Navon, 1977). In the Navon local task, the caffeine effect was not significant: incongruent figures, compared to congruent figures, needed more time to be discriminated both after caffeine $\left(t_{(52)}=-4.411, p<0.0001\right.$; Cohen's $d=-0.606,95 \% \mathrm{CI}=-0.897-$ $\left.-0.31 ; \mathrm{BF}_{10} \geqslant 30\right)$ and placebo intake $\left(t_{(52)}=-2.131, p=0.038\right.$; Cohen's $d=-0.293,95 \% \mathrm{CI}=-0.566--0.016 ; \mathrm{BF}_{10}=1.198$; see Figure 2(b)). In this task, the incongruence effect was not different in the two sessions (caffeine mean $=23 \mathrm{~ms}, \mathrm{SD}=39$; placebo mean $=19 \mathrm{~ms}, \mathrm{SD}=66, t_{(52)}=-0.478, p=0.653$ Cohen's $d=0.065$, $\left.95 \% \mathrm{CI}=-0.205-0.334 ; \mathrm{BF}_{10}=0.167\right)$.

\section{Attentional network task}

The attentional network task was administered to evaluate the effects of caffeine on (a) alerting (the ability to activate a state of vigilance after the appearance of a cue); (b) orienting (the ability to rapidly orient visual attention towards a pre-cued direction); (c) disengagement (the ability to rapidly reorient attention from an uncorrected pre-cued direction); and (d) executive functions (the ability to ignore the presence of incongruent flanker 
Table 3. Attentional abilities. Comparison of caffeine and placebo intake on response time (ms) in the attentional network task: alerting (no cue minus double cue), orienting (double minus valid cue), disengagement (invalid minus valid cue) and executive functions (incongruent minus congruent flanker).

\begin{tabular}{|c|c|c|c|c|c|}
\hline Attentional functions & Caffeine mean (SD) & Placebo mean (SD) & $t$ Test and $p$ value & Cohen's $d$ and 95\% CI & Bayesian factor \\
\hline Alerting (ms) & $18(19)$ & $25(29)$ & $t_{(52)}=-1.624, p=0.11$ & $\begin{array}{l}-0.223 \\
-0.495-0.051\end{array}$ & $\mathrm{BF}_{01}=1.957$ \\
\hline Orienting (ms) & $17(19)$ & $12(19)$ & $t_{(52)}=1.306, p=0.197$ & $\begin{array}{l}0.179 \\
-0.093-0.45\end{array}$ & $\mathrm{BF}_{01}=2.998$ \\
\hline Disengagement (ms) & $23(19)$ & $22(18)$ & $t_{(52)}=0.454, p=0.652$ & $\begin{array}{l}0.062 \\
-0.207-0.332\end{array}$ & $\mathrm{BF}_{01}=6.055$ \\
\hline Executive functions (ms) & $153(69)$ & $153(66)$ & $t_{(52)}=-0.018, p=0.982$ & $\begin{array}{l}-0.002 \\
-0.272-0.267\end{array}$ & $\mathrm{BF}_{01}=6.678$ \\
\hline
\end{tabular}

BF: Bayesian factor; CI: confidence interval; SD: standard deviation.

distractors; Fan et al., 2002; Posner et al., 1984; see Figure 2(a)). Analyses were conducted on corrected choice response time. None of the variables investigated were influenced by caffeine intake (see Figure 2 and Table 3 ).

\section{State anxiety, sleep variables and caffeine in saliva samples}

To evaluate the possible differences in arousal, the state of anxiety was evaluated $30 \mathrm{~min}$ after caffeine/placebo intake (at the beginning of the behavioural assessments). Scores achieved with the questionnaire in the two self-evaluations did not differ between placebo and caffeine intake conditions in both studies (see Table 1).

In addition, the number of hours of sleep, declared before the caffeine or placebo intake evaluations, were not different at the two administration times (see Table 2). The caffeine effect in word text reading speed and in global Navon response times could not be directly attributed to different amounts of sleep before the two (caffeine and placebo) experimental sessions.

Nevertheless, there was a relationship between how the subjects had slept before their caffeine trial compared with their placebo trial and how effective the caffeine was $(r=-0.38, p=0.001$; see Figure 4); that is, the higher their sleep deprivation before the caffeine session the greater their improvement in text reading with caffeine. A small amount of sleep deprivation seems to create a positive effect of caffeine in reading skills. Conversely, a prolonged period of sleep before caffeine intake (in comparison to placebo) results in the beneficial caffeine effect vanishing on text reading speed. But this was not true for the Navon task: incongruent minus congruent response times in the caffeine session minus the same difference in the placebo session and sleep was not significant $(r=-0.09, p=0.541)$.

Salivary caffeine $(\mu \mathrm{g} / \mathrm{mL})$ was significantly different in caffeine and placebo conditions $\left(t_{(52)} 13.048, p=0.0001\right.$, Cohen's $\left.d=1.792,95 \% \mathrm{CI}=1.352-2.225 ; \mathrm{BF}_{10}>30\right)$. Salivary caffeine was higher in the caffeine (mean $=4.93, \mathrm{SD}=1.8)$ than in the placebo (mean=1.48, $\mathrm{SD}=1.11$ ) condition (see Figure 5). Correlational analyses between the difference in salivary caffeine in caffeine and placebo conditions, text reading difference (syll/s), and difference in Navon global task were not significant (all $p \mathrm{~s}>0.46$ ). As shown by Brice and Smith (2001), individual differences of caffeine in saliva samples were not related to individual variations in cognitive performance.

\section{Discussion}

One of the most complex and peculiar abilities of humans is reading. Reading acquisition involves a number of basic cognitive skills, such as visual and phonological abilities, memory and attentional and cross-modal integration abilities (e.g. Bertoni, et al., 2019; Carroll et al., 2016; Chiappe et al., 2002; Franceschini et al., 2012, 2017; Gori et al., 2016; Snowling et al., 2019). Reading also requires the ability to integrate different lexicosemantic information in a global pattern (e.g. Horowitz-Kraus et al., 2014, 2015; see for a meta-analysis Vigneau et al., 2011). The importance of all of these abilities is modulated during the different phases of reading acquisition (Snowling et al., 2019) and their weight is in relation to the specific reading task (HorowitzKraus et al., 2014).

We demonstrated that a small quantity of caffeine specifically enhances text reading speed. The reading speed enhancement induced by caffeine could be measured in comparison to reading development. In particular, the mean improvement in text reading speed obtained after $200 \mathrm{mg}$ of caffeine $(=0.1$ syll/s) was higher than the mean improvement expected in a college student $(=0.083 \mathrm{syll} / \mathrm{s})$ after two months of spontaneous reading development (Stella and Tintoni, 2007). An alternative 'weight' of the transient reading speed improvement could be understood by considering that after consuming $200 \mathrm{mg}$ of caffeine, this article could be read while saving about one minute (see Supplementary Material). In a silent text reading task, Flory and Gilbert (1943), comparing the effects of about 300 $\mathrm{mg}$ of caffeine, $15 \mathrm{mg}$ of benzedrine sulphate and a placebo pill, showed that, despite each substance appearing to increase reading performance, these reading speed improvements were not significant. Their results, in light of our data, could be justified by their reduced sample size.

Word and pseudoword reading speed were not accelerated after caffeine intake, suggesting that text reading speed improvement could not be due to a faster access to sublexical and lexical phonological representation of the written words composing the text. Similarly, we did not observe improvements in phonological memory or in the rapid automatised naming, confirming that these cognitive skills could also not be linked to caffeine's effect on text reading speed. Left temporo-parietal phonological skills (Vigneau et al., 2011) are strongly linked to reading acquisition and development (Snowling et al., 2019), but these skills do not appear to be enhanced by caffeine. 


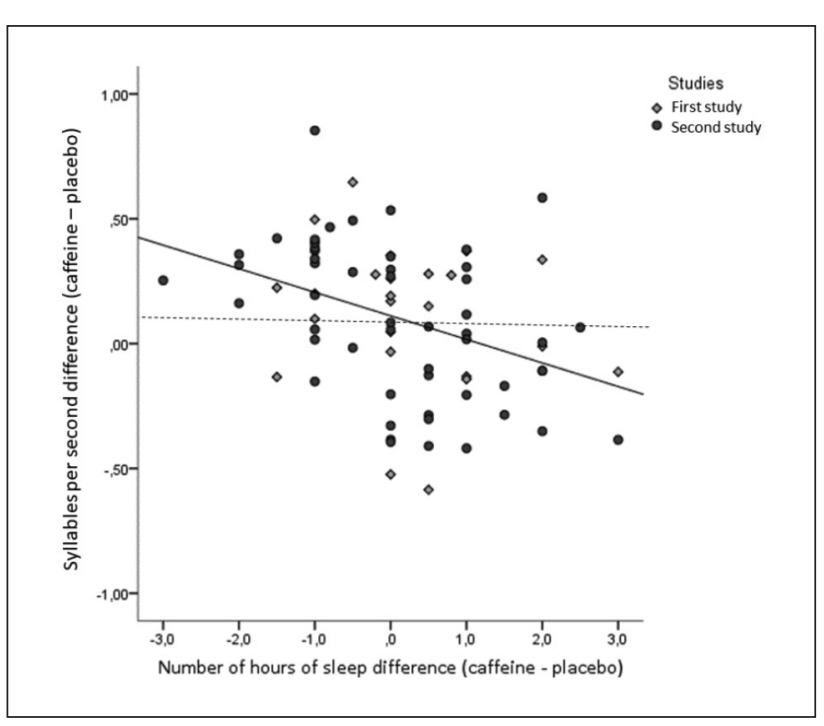

Figure 4. Reading improvement and sleep deprivation. Correlation between difference in syllables for second (performance in caffeine minus placebo), and the difference of sleep hours (hours of sleep before caffeine minus placebo condition). The solid line represents the correlation, between variables, whereas the dashed line represents the mean improvement in text reading speed.

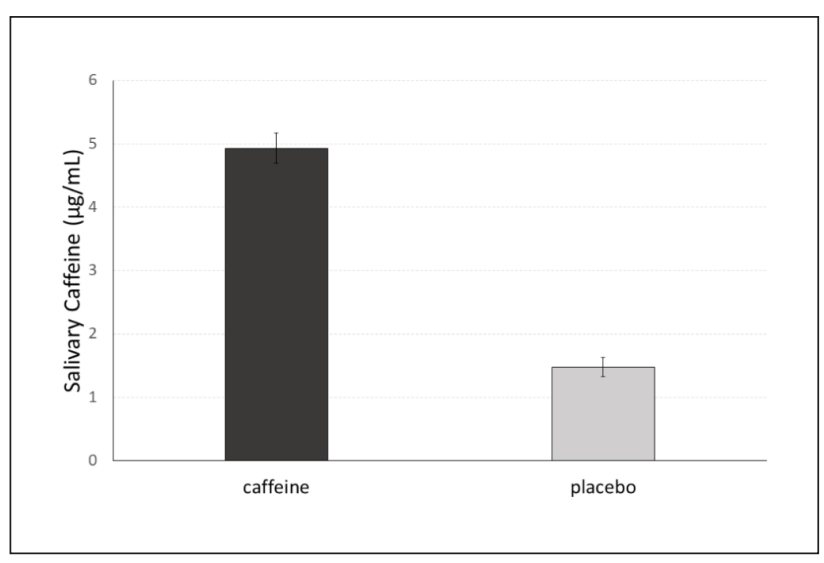

Figure 5. Salivary caffeine $(\mu \mathrm{g} / \mathrm{mL})$ in saliva samples collected at the beginning of tasks administration in caffeine and placebo conditions. Error bars represent standard error mean.

We found a selective effect on text reading speed in line with the literature that suggests a critical role of right inferior frontal gyrus and right fasciculus arcuatus in sentence completion, but not with single-word reading tasks (Horowitz-Kraus et al., 2014). In line with our results, greater access to semantic information after caffeine intake has already been shown in the literature. Caffeine could produce an improvement of semantic abilities, probably by altering central noradrenergic functioning, whereas improvements in logical reasoning and encoding of new information appear linked to the effects on the cholinergic system (Smith et al., 1994, 2003). Different results should be expected in developing brains of children with reading difficulties. In this case, the same neurocognitive network could be involved in non-automatised single-word or syllable decoding. Additional attentional deployment could be involved in the analysis of the global patterns of letters that compose words or syllables (Franceschini et al., 2017; Hoeft et al., 2011).

Alerting and executive attentional functions could influence reading performance (Shaywitz et al., 2017), but after $200 \mathrm{mg}$ caffeine intake we did not observe changes in these skills or in orienting. Results of the effects of caffeine on the different components of the attentional network task are controversial (e.g. Einöther and Giesbrecht, 2013; Nehlig et al., 1992; Nehlig, 2004). The null effects of caffeine on lexical and sublexical reading routes, as well as on engagement and disengagement mechanisms of visual attention, are in line with some studies suggesting a possible relationship between the visual attentional orienting network and word and pseudoword reading acquisition (e.g. Ekstrand, et al., 2019; Facoetti et al., 2010). However, we could not exclude the possibility that a larger quantity of caffeine could also produce observable effects in these cognitive skills.

Performance in the perceptual Navon task shows that caffeine improves global pattern perception, reducing the interference of local stimuli. A similar result was shown by Mahoney et al. (2011), in which different quantities of caffeine - with an asymptotic effect at about $200 \mathrm{mg}$ - were able to improve global processing in a similar Navon task using local and global letters stimuli.

Global pattern perception and text reading abilities appear causally linked in a developing brain (Franceschini et al., 2017). The improvements in text reading speed and global perception do not appear to be linearly connected, however, the improvements in reading and the reduction of local interference during global processing are categorically associated in the present study. The different relationship between global pattern perception and reading speed shown in pre-readers and undergraduate students could be sought in cognitive developmental mechanisms in which visuoperceptual skills might be linearly related to reading acquisition only during the early developmental stage (Snowling et al., 2019).

Our exploratory analysis of the possible different effects of caffeine on males and females seems to confirm larger effects in males compared to females (Adan et al., 2008). Deficit in global precedence extraction has been described in several neurodevelopmental disorders: developmental dyslexia (Franceschini et al., 2017), attention deficit and hyperactivity disorder (Song and Hakoda, 2012) and autism spectrum disorder (Van der Hallen et al., 2015). Considering that these neurodevelopmental disorders are more frequent in males (American Psychiatric Association, 2013), caffeine appears to be an interesting candidate for intervention training. However, our study presents some limitations, such as the unbalanced number of males and females, the use of a unique quantity of caffeine and the absence of a fine semantic task. A larger and balanced sample of participants with and without these developmental disorders will be necessary to confirm the observed gender differences and to pave the way for possible future applications.

Our results show that the effect of caffeine on meaningful word text reading speed was boosted by a small amount of sleep deprivation. Indeed, if in the previous night one loses two hours of sleep, then the savings when reading this article will be more than doubled. Caffeine intake results in different effects in cases of sleep loss (Caldwell et al., 2008; McLellan et al., 2016; Smith, 2011; Weiss and Laties, 1962), and in the absence of sleep deprivation caffeine can impair performance (McLellan et al., 2016). Indeed, Linde (1994), in a logical reasoning task, showed that 
caffeine intake produced improvement in a verbal sentence comprehension and spatial relationship between items reproduction task after sleep deprivation, whereas after normal sleep a slight worsening was observed. The correlation found between reading speed improvement and quantity of sleep provides evidence of the possible effect of caffeine on specific neurotransmitters linked to changes in the sleep/waking cycle (Longordo et al., 2009). Serotonin levels - conditioned by caffeine intake - can influence visual perception and text reading speed. Sleep deprivation stimulates serotonin release (Grossman et al., 2000), and this neurotransmitter is largely present in the medio- and orbitofrontal areas of the right hemisphere (Arato et al., 1991). Interestingly, it has been demonstrated that one night of sleep deprivation before the administration of a computerised Navon task negatively influenced the activation of the inferior occipital cortex and the inferior frontal gyrus, only when the contrast of the stimuli decreased (Chee and Tan, 2010), a condition that mainly involves magnocellular-dorsal pathway activity. It is possible that reading and global visual processing are not directly connected to each other, but that both networks underlie the same neurotransmitter system. Nevertheless, further studies are necessary to understand which neural pathways and neuromodulators regulate the influence of caffeine on cognitive functions.

Thus, caffeine improves text reading speed mainly after a small amount of sleep deprivation, and this improvement is associated with a specific enhancement in the extraction of visual global pattern information. Changes in perceptual processing could be the basis of changes in conceptual processing (Mesulam, 1990; Van Dantzig et al., 2008). The activation of fronto-parietal areas could be a part of the network associated with the observed perceptual and reading enhancements (Serra-Grabulosa et al., 2010).

\section{Acknowledgements}

The following author contributions were made: conceptualization SF, SB, AF; methodology SF, AF; software SF; formal analysis SF, ML; investigation MM, GP; writing original draft SF; supervision SF, AF, AA, SG; project administration $\mathrm{SF}, \mathrm{SB}$; funding acquisition $\mathrm{SF}, \mathrm{AF}, \mathrm{ML}, \mathrm{SB}$.

\section{Declaration of conflicting interests}

The author(s) declared no potential conflicts of interest with respect to the research, authorship, and/or publication of this article.

\section{Funding}

The author(s) disclosed receipt of the following financial support for the research, authorship, and/or publication of this article: This work was funded by a grant from MIUR (Dipartimenti di Eccellenza DM 11/05/2017 n.262) to the Department of General Psychology, University of Padua to SF, and the CARIPARO Foundation (Borse di Dottorato CARIPARO 2015, to SB).

\section{ORCID iD}

Sandro Franceschini (iD https://orcid.org/0000-0002-2326-6800

\section{References}

Adan A, Prat G and Sànchez-Turet M (2008) Early effects of caffeinated and decaffeinated coffee on subjective state and gender differences. Prog Neuropsychopharmacol Biol Psychiatry 32: 1698-1703.

American Psychiatric Association (2013) Diagnostic and Statistical Manual of Mental Disorders (DSM-5®). 5th ed. Arlington, VA: American Psychiatric Publishing, Inc.
Arato M, Frecska E, MacCrimmon DJ, et al. (1991) Serotonergic interhemispheric asymmetry: Neurochemical and pharmaco-EEG evidence. Prog Neuropsychopharmacol Biol Psychiatry 15: 759-764.

Barone JJ and Roberts HR (1996) Caffeine consumption. Food Chem Toxicol 34: 119-129.

Bertoni S, Franceschini S, Ronconi L, et al. (2019) Is excessive visual crowding causally linked to developmental dyslexia? Neuropsychologia 130: 107-117.

Breznitz Z, Shaul S, Horowitz-Kraus T, et al. (2013) Enhanced reading by training with imposed time constraint in typical and dyslexic adults. Nat Commun 4: 1486.

Brice CF and Smith AP (2001) Caffeine levels in saliva: Associations with psychosocial factors and behavioural effects. Hum Psychopharmacol 16: 507-521.

Brunyé TT, Mahoney CR, Rapp DN, et al. (2012) Caffeine enhances real-world language processing: Evidence from a proofreading task. J Exp Psychol Appl 18: 95-108.

Caldwell JA, Caldwell JL and Schmidt RM (2008) Alertness management strategies for operational contexts. Sleep Med Rev 12: 257-273.

Carroll JM, Solity J and Shapiro L R (2016) Predicting dyslexia using prereading skills: The role of sensorimotor and cognitive abilities. $J$ Child Psychol Psychiatry 57: 750-758.

Chee MW and Tan JC (2010) Lapsing when sleep deprived: Neural activation characteristics of resistant and vulnerable individuals. Neuroimage 51: 835-843.

Chiappe P, Siegel LS and Wade-Woolley L (2002) Linguistic diversity and the development of reading skills: A longitudinal study. Sci Stud Read 6: 369-400.

Costanzo F, Menghini D, Caltagirone C, et al. (2012) High frequency rTMS over the left parietal lobule increases non-word reading accuracy. Neuropsychologia 50: 2645-2651.

Dehaene S, Cohen L, Morais J, et al. (2015) Illiterate to literate: Behavioural and cerebral changes induced by reading acquisition. Nat Rev Neurosci 16: 234-244.

Delis DC, Robertson LC and Efron R (1986) Hemispheric specialization of memory for visual hierarchical stimuli. Neuropsychologia 24 : 205-214.

Doherty M and Smith PM (2005) Effects of caffeine ingestion on rating of perceived exertion during and after exercise: A meta-analysis. Scand J Med Sci Spor 15: 69-78.

Einöther SJ and Giesbrecht T (2013) Caffeine as an attention enhancer: Reviewing existing assumptions. Psychopharmacology 225: 251274.

Ekstrand C, Neudorf J, Gould L, et al. (2019) Where words and space collide: The overlapping neural activation of lexical and sublexical reading with voluntary and reflexive spatial attention. Brain Res 1706: $1-12$.

Facoetti A, Trussardi AN, Ruffino M, et al. (2010) Multisensory spatial attention deficits are predictive of phonological decoding skills in developmental dyslexia. J Cognitive Neurosci 22: 1011-1025.

Fan J, McCandliss BD, Sommer T, et al. (2002) Testing the efficiency and independence of attentional networks. J Cognitive Neurosci 14 : $340-347$.

Ferré S (2008) An update on the mechanisms of the psychostimulant effects of caffeine. J Neurochem 105: 1067-1079.

Flory CD and Gilbert J (1943) The effects of benzedrine sulphate and caffeine citrate on the efficiency of college students. J Appl Psychol 27: $121-134$.

Franceschini S, Bertoni S, Gianesini T, et al. (2017) A different vision of dyslexia: Local precedence on global perception. Sci Rep 7: 17462.

Franceschini S, Bertoni S, Ronconi L, et al. (2016) "Batteria De.Co.Ne. per la lettura" strumenti per la valutazione delle abilità di lettura nelle scuole primarie di primo grado. Dislessia 13: 319-347.

Franceschini S, Gori S, Ruffino M, et al. (2012) A causal link between visual spatial attention and reading acquisition. Curr Biol 22: 814-819. 
Fredholm BB, Bättig K, Holmén J, et al. (1999) Actions of caffeine in the brain with special reference to factors that contribute to its widespread use. Pharmacol Rev 51: 83-133.

Gori S, Seitz AR, Ronconi L, et al. (2016) Multiple causal links between magnocellular-dorsal pathway deficit and developmental dyslexia. Cereb Cortex 26: 4356-4369.

Green CS, Bavelier D, Kramer AF, et al. (2019) Improving methodological standards in behavioral interventions for cognitive enhancement. $J$ Cogn Enhanc 3: 2-29.

Grossman GH, Mistlberger RE, Antle MC, et al. (2000) Sleep deprivation stimulates serotonin release in the suprachiasmatic nucleus. Neuroreport 11: 1929-1932.

Hancock R, Pugh KR and Hoeft F (2017) Neural noise hypothesis of developmental dyslexia. Trends Cogn Sci 21: 434-448.

Heckman MA, Weil J and De Mejia EG (2010) Caffeine (1,3,7-trimethylxanthine) in foods: A comprehensive review on consumption, functionality, safety, and regulatory matters. J Food Sci 75: R77-R87.

Hochstein S and Ahissar M (2002) View from the top: Hierarchies and reverse hierarchies in the visual system. Neuron 36: 791-804.

Hoeft F, McCandliss BD, Black JM, et al. (2011) Neural systems predicting long-term outcome in dyslexia. Proc Natl Acad Sci U S A 108: 361-366.

Horowitz-Kraus T, Grainger M, DiFrancesco M, et al. (2015) Right is not always wrong: DTI and fMRI evidence for the reliance of reading comprehension on language-comprehension networks in the right hemisphere. Brain Imaging Behav 9: 19-31.

Horowitz-Kraus T, Vannest JJ, Kadis D, et al. (2014) Reading acceleration training changes brain circuitry in children with reading difficulties. Brain Behav 4: 886-902.

Judica A and De Luca M (1993) Prova di velocità di lettura brani per la scuola media superiore. Available at: https://www.hsantalucia.it/ sites/default/files/fsl_labdislessia_lettura_brani_test.pdf(accessed 25 September 2019).

Kauffmann L, Ramanoël S and Peyrin C (2014) The neural bases of spatial frequency processing during scene perception. Front Integr Neurosci 8: 37.

Lazzari R and Pancheri P (1980) STAI Questionario di autovalutazione dell'ansia di stato e di tratto. Firenze: Organizzazioni Speciali.

Linde L (1994) An auditory attention task: A note on the processing of verbal information. Percept Motor Skill 78: 563-570.

Longordo F, Kopp C and Lüthi A (2009) Consequences of sleep deprivation on neurotransmitter receptor expression and function. Eur $J$ Neurosci 29: 1810-1819.

Mahoney CR, Brunyé TT, Giles G, et al. (2011) Caffeine-induced physiological arousal accentuates global processing biases. Pharmacol Biochem Behav 99: 59-65.

Martinez A, Moses P, Frank L, et al. (1997) Hemispheric asymmetries in global and local processing: Evidence from fMRI. Neuroreport 8: $1685-1689$.

McLellan TM, Caldwell JA and Lieberman HR (2016) A review of caffeine's effects on cognitive, physical and occupational performance. Neurosci Biobehav Rev 71: 294-312.

Mesulam MM (1990) Large-scale neurocognitive networks and distributed processing for attention, language, and memory. Ann Neurol 28: 597-613.

Navon D (1977) Forest before trees: The precedence of global features in visual perception. Cogn Psychology 9: 353-383.

Nehlig A (2004) Coffee, Tea, Chocolate, and the Brain. Boca Raton, FL: CRC Press.

Nehlig A, Daval JL and Debry G (1992) Caffeine and the central nervous system: Mechanisms of action, biochemical, metabolic and psychostimulant effects. Brain Res Rev 17: 139-170.

Peters JL, De Losa L, Bavin EL, et al. (2019) Efficacy of dynamic visuoattentional interventions for reading in dyslexic and neurotypical children: A systematic review. Neurosci Biobehav Rev 100: 58-76.
Posner MI, Walker JA, Friedrich FJ, et al. (1984) Effects of parietal injury on covert orienting of attention. J Neurosci 4: 1863-1874.

Proverbio AM, Minniti A and Zani A (1998) Electrophysiological evidence of a perceptual precedence of global vs. local visual information. Cogn Brain Res 6: 321-334.

Ronconi L, Basso D, Gori S, et al. (2012) TMS on right frontal eye fields induces an inflexible focus of attention. Cereb Cortex 24: 396-402.

Ruxton CHS (2008) The impact of caffeine on mood, cognitive function, performance and hydration: A review of benefits and risks. Nutr Bull 33: $15-25$.

Sergent J (1982) The cerebral balance of power: Confrontation or cooperation? J Exp Psychol Hum Percept Perform 8: 253-272.

Serra-Grabulosa JM, Adan A, Falcón C, et al. (2010) Glucose and caffeine effects on sustained attention: An exploratory fMRI study. Hum Psychopharmacol 25: 543-552.

Shaywitz S, Shaywitz B, Wietecha L, et al. (2017) Effect of atomoxetine treatment on reading and phonological skills in children with dyslexia or attention-deficit/hyperactivity disorder and comorbid dyslexia in a randomized, placebo-controlled trial. J Child Adol Psychop 27: $19-28$.

Smith A (2002) Effects of caffeine on human behavior. Food Chem Toxicol 40: 1243-1255.

Smith AP (2011) Caffeine: Practical implications. In: Kanarek RB and Lieberman HR (eds) Diet, Brain, Behavior: Practical Implications. Taylor \& Francis, pp. 271-292.

Smith A, Brice C, Nash J, et al. (2003) Caffeine and central noradrenaline: Effects on mood, cognitive performance, eye movements and cardiovascular function. J Psychopharmacol 17: 283-292.

Smith A, Kendrick A, Maben A, et al. (1994) Effects of breakfast and caffeine on cognitive performance, mood and cardiovascular functioning. Appetite 22: 39-55.

Snowling MJ, Nash HM, Gooch DC, et al. (2019) Developmental outcomes for children at high risk of dyslexia and children with Developmental Language Disorder. Child Dev. Epub ahead of print 24 January 2019. DOI: 10.1111/cdev.13216.

Song Y and Hakoda Y (2012) The interference of local over global information processing in children with attention deficit hyperactivity disorder of the inattentive type. Brain Dev 34: 308-317.

Stella G and Tintoni C (2007) Indagine e rilevazione sulle abilità di lettura nelle scuole secondarie di secondo grado. Dislessia 4: 271-285.

Van Dantzig S, Pecher D, Zeelenberg R, et al. (2008) Perceptual processing affects conceptual processing. Cogn Sci 32: 579-590.

Van der Hallen R, Evers K, Brewaeys K, et al. (2015) Global processing takes time: A meta-analysis on local-global visual processing in ASD. Psychol Bull 141: 549-573.

Vigneau M, Beaucousin V, Hervé PY, et al. (2011) What is righthemisphere contribution to phonological, lexico-semantic, and sentence processing? Insights from a meta-analysis. Neuroimage 54: 577-593.

Weiss B and Laties VG (1962) Enhancement of human performance by caffeine and the amphetamines. Pharmacol Rev 14: 1-36.

Weissman DH and Banich MT (1999) Global-local interference modulated by communication between the hemispheres. $J$ Exp Psychol Gen 128: 283-308.

Weissman DH, Roberts KC, Visscher KM, et al. (2006) The neural bases of momentary lapses in attention. Nat Neurosci 9: 971-978.

Weissman DH and Woldorff MG (2004) Hemispheric asymmetries for different components of global/local attention occur in distinct temporo-parietal loci. Cereb Cortex 15: 870-876.

Yamaguchi S, Yamagata S and Kobayashi S (2000) Cerebral asymmetry of the "top-down" allocation of attention to global and local features. J Neurosci 20: RC72-RC72.

Zago L, Petit L, Turbelin MR, et al. (2008) How verbal and spatial manipulation networks contribute to calculation: An fMRI study. Neuropsychologia 46: 2403-2414. 